\title{
Interband transitions and core excitation in highly oriented pyrolytic graphite studied by inelastic synchrotron x-ray scattering: Band-structure information
}

\author{
W. Schülke, U. Bonse, H. Nagasawa, A. Kaprolat, and A. Berthold \\ Institute of Physics, University of Dortmund, D-4600 Dortmund 50, Federal Republic of Germany
}

(Received 12 August 1987; revised manuscript received 17 February 1988)

\begin{abstract}
The dynamic structure factor $S(\mathbf{q}, \omega)$ of electrons in highly oriented pyrolytic graphite for q\|c and $\mathrm{q} \perp \mathrm{c}$ with $0.37<q<2.06$ a.u. was measured at $0.8 \mathrm{eV}$ resolution by inelastic scattering of synchrotron $x$ radiation. The energy-transfer regions both of interband transitions and of core excitations were investigated. By means of Kramers-Kronig transformation $\operatorname{Re}[\epsilon(\mathbf{q}, \omega)]$ and $\operatorname{Im}[\epsilon(\mathbf{q}, \omega)]$ were obtained, where peaks of the latter were attributed directly to maxima of projections off the joint density of states achieved by both dipole selection rules and $q$-dependent matrix elements. Making use of both $\mathrm{x}$-ray emission and photoemission data, limits for the energy positions of some conduction bands could be established by means of the $\operatorname{Im}[\epsilon(\mathbf{q}, \omega)]$ data. As far as weakly- $k_{z}$ dispersing bands are concerned, their energy positions were found to be in good agreement with recent band-structure calculations. On the other hand, the measured energy ranges of the bands that could be identified as belonging to the recently proposed interlayer states disagree with most of the relevant band-structure calculations, though they are not far from the results of other spectroscopies. The strict bulk origin of the inelastic-scattering-based band-structure information obtained is stressed. The experimental data are also discussed in view of the interpretation of previous lowresolution inelastic-scattering data in terms of correlation-induced fine structure for $\mathrm{q} \perp \mathbf{c}$.
\end{abstract}

\section{INTRODUCTION}

Graphite, the archetypal layered structure, is one of the most thoroughly studied solids. As first exhibited by two-dimensional (2D) band-structure calculations, ${ }^{1-7}$ the following order of bands is now commonly accepted: With increasing energy, the bonding $\sigma$ state is followed by a bonding $\pi$ state, both forming the valence band, whereas the sequence in the conduction band is $\pi^{*}$ and $\sigma^{*}$ antibonding. The sequence is due to the much stronger intralayer covalent interaction between the $2 s$, $2 p_{x}$, and $2 p_{y}$ atomic orbitals ( $s p^{2}$ hybrids), from which the $\sigma$ states are derived, compared with the intralayer interaction of the $p_{z}$ orbitals, which form the $\pi$ states. The graphite bands exhibit a strong paraboliclike dispersion in the $k_{x}$ and $k_{y}$ directions. The dispersion in the $k_{z}$ direction, as predicted by parametrized ${ }^{1,3,5}$ threedimensional (3D) band-structure calculations, is generally weak, due to the very weak interlayer interaction. However, recent calculations ${ }^{8-10}$ have revealed a strongly- $k_{z}$ dispersing state in the conduction band with large electron density between the carbon layers (interlayer state). This state seems to be of crucial importance for the understanding of donor-type intercalation compounds of graphite. ${ }^{10}$

If we neglect for the moment all studies concerning Fermi-surface parameters and transport-related phenomena, we can divide the experimental techniques, devoted so far to the band structure of graphite, into three categories:

(i) First, there are techniques that provide direct information about the occupied valence band, such as $\mathrm{x}$-ray photoemission (XPS), ${ }^{11}$ and ultraviolet photoemission
(UPS), ${ }^{12}$ angle-resolved photoemission (ARPES), ${ }^{13-18}$ angle-resolved $\mathrm{x}$-ray emission spectroscopy (ARXES), ${ }^{19}$ and, via the band-structure wave functions, real-space density ${ }^{20}$ and momentum-space density ${ }^{21}$ measurements. The results of all these experiments have offered a consistent picture of the valence-band structure of graphite, as far as bandwidth, band position, and band dispersion are concerned. All features of the experimental valenceband structure are in good agreement with the calculations of Refs. 22, 8, and 9. Certainly there exists a tendency of the calculation to underestimate the total bandwidth. On the other hand, most of the results of Ref. 23 were ruled out by the experiments.

(ii) The second of the above-mentioned categories consists of techniques that provide information about the unoccupied conduction bands, like angle-resolved inverse photoemission (ARIPS), ${ }^{24-26}$ constant-final-state spectroscopy (CFSPES), ${ }^{27}$ excited secondary-electron emission (SEES), ${ }^{22,28,29}$ angle-resolved total-current spectroscopy (TCS), ${ }^{26,30}$ secondary-electron features of ARPES, ${ }^{15-18} K$-edge spectroscopy by using both soft-xray absorption (SXAS) $)^{31,32}$ and electron-energy-loss spectroscopy (EELS), ${ }^{28,33,34}$ soft-x-ray appearance-potential spectroscopy (SXAPS), ${ }^{35}$ and bremsstrahlung isochromat spectroscopy (BIS). ${ }^{35}$ In spite of this considerable effort, a consistent experimentally based picture of the conduction-band structure of graphite is still lacking. The experiments differ significantly as regards the energy position of certain features and their assignment to details of the theoretical conduction-band structure. The statement applies in particular to the energy position and dispersion of the interlayer state already mentioned above. The discrepancies are mainly due to uncertainties with the interpretation of experimental data of TCS and 
SEES, but also of ARPES and ARIPS, as far as surface states are involved. Furthermore, there does not exist a commonly accepted theoretical picture of the graphite band structure that could serve as a guide to the correct interpretation of the large variety of experimental data.

(iii) For this reason the third category of experimental techniques will become more important, namely those that aim to measure the joint density of states (JDOS) between valence and conduction bands by exciting interband transitions. Thus, based on the experimentally well-known valence-band structure of graphite, in principle, the conduction-band structure should be deducible. This category consists of all types of optical measurements, ${ }^{36-41}$ which were rather helpful to fix certain transitions within the band structure, together with EELS, ${ }^{28,42-44}$ that offers additionally the insight into nonvertical interband transitions to a certain extent.

It is the aim of this paper to introduce inelastic $x$-rayscattering spectroscopy (IXSS) as a tool for investigating both the symmetry-projected density of states (DOS) of graphite by core excitation, and the symmetry-projected JDOS by exciting interband transition. IXSS has become competitive with other spectroscopies by utilizing the advantages of synchrotron $x$ rays. Thus an energy resolution of $1 \mathrm{eV}$ and a signal-to-background ratio of better than 100 could be achieved. So far, only a low-resolution IXSS measurement of graphite using a conventional $x$-ray source has been published. ${ }^{45}$ The main advantages of IXSS over SXAS and EELS consist of the following:

(i) Both the small magnitude of the double-differential cross section of inelastic x-ray scattering compared to that of inelastic electron scattering and its $q^{2}$ dependence ( $q$ is defined as transferred momentum) make multiple scattering much less troublesome. Thus, a much larger range of $q$ values can be provided than is available with EELS. Furthermore, a thorough application of the Kramers-Kronig transformation (KKT) of the experimental data becomes feasible.

(ii) The experimental information of IXSS is derived from the bulk. The influence of the surface and of surface contaminations can be neglected completely.

(iii) Typical sample dimensions for IXSS are of the order of millimeters so that single-crystal preparation for any direction of $q$ is possible without difficulty.

(iv) In the case of IXSS, the direction and magnitude of $q$ are not coupled by experimental constraints as in EELS, so that symmetry-induced features can be read from each spectrum separately and selection rules can be exploited without limitations.

By utilizing these advantages and by relying on the well-established experimental valence-band structure of graphite, we have derived symmetry-projected information about the conduction-band structure. After a short survey in Sec. II A of relevant principles of IXSS, some experimental details are given in Sec. II B. In Sec. III the experimental IXSS results on graphite are presented both for core excitation (Sec. III A) and for excitation of interband transitions (Sec. III $B$ for $q \| c$ and Sec. III $C$ for $q \perp c)$. The discussion of the results based on the comparison with both recent band-structure calculations and the results of other spectroscopies will follow in Sec. IV, where the sequence of presentation is the same as in Sec. III. Finally, Sec. V contains concluding remarks.

\section{INELASTIC X-RAY SCATTERING}

\section{A. Principles}

By treating the scattering process in first-order perturbation theory, the double-differential scattering cross section (DDSCS) can be directly related to the dynamic structure factor (DSF), $S(\mathrm{q}, \omega)$, of the scattering electron system via $^{46}$

$$
\frac{d^{2} \sigma}{d \Omega d \omega}=\left(\mathbf{e}_{0} \cdot \mathbf{e}^{\prime}\right)^{2} r_{0}^{2}\left(\omega^{\prime} / \omega_{0}\right) S(\mathbf{q}, \omega),
$$

where $\hbar \omega \equiv \hbar\left(\omega_{0}-\omega^{\prime}\right)$ is the energy and $\hbar \mathbf{q}=\hbar\left(\mathbf{K}_{0}-\mathbf{K}^{\prime}\right)$ is the momentum transferred to the scattering system, if a photon of energy $\hbar \omega_{0}$, momentum $\hbar \mathbf{K}_{0}$, and polarization vector $e_{0}$ is scattered into a photon, characterized by the corresponding quantities $\hbar \omega^{\prime}$ and $\hbar \mathbf{K}^{\prime}$, and $\mathbf{e}^{\prime} \cdot r_{0}$ is the classical electron radius. $S(\mathbf{q}, \omega)$ is connected with the dielectric response function (DRF) $\epsilon^{-1}(\mathbf{q}, \omega)$ by means of the fluctuation-dissipation theorem ${ }^{47}$

$$
S(\mathrm{q}, \omega)=-\left(\hbar q^{2} / 4 \pi^{2} e^{2} n\right) \operatorname{Im}\left[\epsilon^{-1}(\mathrm{q}, \omega)\right],
$$

where $n$ is the electron density,

If we neglect all microscopic local-field effects ${ }^{48}$ caused by the periodic crystal structure of a solid, we have, within the limits of the random-phase approximation (RPA), the following expression for the DRF:

$$
\operatorname{Im}\left[\epsilon^{-1}(\mathbf{q}, \omega)\right]=-\epsilon_{2}(\mathbf{q}, \omega) /\left[\epsilon_{1}^{2}(\mathbf{q}, \omega)+\epsilon_{2}^{2}(\mathbf{q}, \omega)\right],
$$

where $\epsilon(\mathbf{q}, \omega) \equiv \epsilon_{1}(\mathbf{q}, \omega)+i \epsilon_{2}(\mathbf{q}, \omega)$ is given by ${ }^{49}$

$$
\begin{aligned}
\epsilon(\mathbf{q}, \omega)=1+\lim _{\eta \rightarrow 0} \frac{4 \pi e^{2}}{q^{2}} \sum_{\substack{\mathbf{k}, l \\
\mathbf{k}^{\prime}, l^{\prime}}} \frac{\left|\left\langle\mathbf{k}^{\prime}, l^{\prime}\left|e^{i \mathbf{q} \cdot \mathbf{r}}\right| \mathbf{k}, l\right\rangle\right|^{2}}{\hbar \omega+E(\mathbf{k}, l)-E\left(\mathbf{k}^{\prime}, l^{\prime}\right)+i \eta} \\
\times\left[f_{0}\left(\mathbf{k}^{\prime}, l^{\prime}\right)-f_{0}(\mathbf{k}, l)\right] .
\end{aligned}
$$

In this relation the electron states of the scattering system are represented by one-electron Bloch states with wave vector $\mathbf{k}$, band index $l$, energy $E(\mathbf{k}, l)$, and occupation number $f_{0}(\mathbf{k}, l)$.

The band-structure information, obtainable from IXSS, is represented in its most elementary form by $\epsilon_{2}(\mathbf{q}, \omega)$, the imaginary part of the dielectric function (DF):

$$
\begin{aligned}
\epsilon_{2}(\mathbf{q}, \omega)=-\frac{4 \pi^{2} e^{2}}{q^{2}} \sum_{\substack{\mathbf{k}, l \\
\mathbf{k}^{\prime}, l^{\prime}}}\left|\left\langle\mathbf{k}^{\prime}, l^{\prime}\left|e^{i \mathbf{q} \cdot \mathbf{r}}\right| \mathbf{k}, l\right\rangle\right|^{2} \\
\times\left[f_{0}\left(\mathbf{k}^{\prime}, l^{\prime}\right)-f_{0}(\mathbf{k}, l)\right] \\
\times \delta\left(\hbar \omega+E(\mathbf{k}, l)-E\left(\mathbf{k}^{\prime}, l^{\prime}\right)\right) .
\end{aligned}
$$

According to Eq. (5), $\epsilon_{2}$ reflects the JDOS via the $\delta$ function weighted by the squared matrix element 
$\left\langle\mathbf{k}^{\prime}, l^{\prime}\left|e^{i \mathbf{q} \cdot \mathbf{r}}\right| \mathbf{k}, l\right\rangle$, that can produce symmetry projections of the JDOS.

The DF can be extracted from the measured spectral distribution (SD) of the radiation, scattered with a welldefined momentum transfer $\mathbf{q}$, in the following way.

If one can neglect multiple scattering, the measured $\mathrm{SD}$ is directly related to $S(\mathrm{q}, \omega)$ via Eq. (1) and can be brought to an absolute scale by means of the $f$ sum rule:

$$
\int_{0}^{\infty} S(\mathbf{q}, \omega) \omega d \omega=\hbar q^{2} / 2 m \text {. }
$$

Absolute values of $S(\mathbf{q}, \omega)$ can be transformed into absolute values of $-\operatorname{Im}\left[\epsilon^{-1}(q, \omega)\right]$ by means of Eq. (2). The latter can be subjected to a Kramers-Kronig transformation in order to get $\operatorname{Re}\left[\epsilon^{-1}(\mathbf{q}, \omega)\right]$ by using the wellknown relation

$$
\begin{aligned}
\operatorname{Re}\left[\epsilon^{-1}(\mathbf{q}, \omega)\right]=1+ & {\left[\frac{2}{\pi}\right] } \\
& \times \int \omega^{\prime} d \omega^{\prime} \operatorname{Im}\left[\epsilon^{-1}\left(\mathbf{q}, \omega^{\prime}\right)\right] /\left(\omega^{\prime 2}-\omega^{2}\right) .
\end{aligned}
$$

Finally $\epsilon_{1}(\mathbf{q}, \omega)$ and $\epsilon_{2}(\mathbf{q}, \omega)$ can be obtained by using

$$
\begin{aligned}
& \epsilon_{1}(\mathbf{q}, \omega)=\operatorname{Re}\left[\epsilon^{-1}(\mathbf{q}, \omega)\right] /(\left\{\operatorname{Re}\left[\epsilon^{-1}(\mathbf{q}, \omega)\right]\right\}^{2} \\
&+\left.\left\{\operatorname{Im}\left[\epsilon^{-1}(\mathbf{q}, \omega)\right]\right\}^{2}\right), \\
& \epsilon_{2}(\mathbf{q}, \omega)=-\operatorname{Im}\left[\epsilon^{-1}(\mathbf{q}, \omega)\right] /\left(\left\{\operatorname{Re}\left[\epsilon^{-1}(\mathbf{q}, \omega)\right]\right\}^{2}\right. \\
&\left.+\left\{\operatorname{Im}\left[\epsilon^{-1}(\mathbf{q}, \omega)\right]\right\}^{2}\right) .
\end{aligned}
$$

According to Eq. (5), peaks in $\epsilon_{2}(q, \omega)$ can be directly related to peaks in the symmetry-projected JDOS. Finite values of $\mathbf{q}$ also admit nonvertical interband transitions, thus opening an additional degree of freedom for probing of the band structure.

A further selection among transitions that contribute to peaks of the JDOS can be provided by the $q$ dependence of the transition matrix element (TME), especially transitions to final states of graphite, the wave functions of which are free-electron-like are concerned. Let the final-state wave function be approximated by

$$
\psi_{\mathbf{k}^{\prime}, l^{\prime}}(\mathbf{r})=V^{-1 / 2} e^{i \mathbf{p}^{\prime} \cdot \mathbf{r}}
$$

with

$$
\begin{aligned}
\mathbf{p}^{\prime}=\mathbf{p}_{\perp}^{\prime}+\mathbf{p}_{\|}^{\prime}= & \left\{2 m\left[E\left(\mathbf{k}_{\perp}^{\prime}, l^{\prime}\right)-E_{\perp}\right]\right\}^{1 / 2} \hat{\mathbf{k}}_{\perp}^{\prime} / \hbar \\
& +\left\{2 m\left[E\left(\mathbf{k}_{\|}^{\prime}, l^{\prime}\right)-E_{\|}\right]\right\}^{1 / 2} \widehat{\mathbf{k}}_{\|}^{\prime} / \hbar,
\end{aligned}
$$

and $E_{\perp}$ and $E_{\|}$appropriately chosen.

Equation (11) takes into account that the dispersion behavior of $E\left(\mathbf{k}^{\prime}, l^{\prime}\right)$ is different for $\mathbf{k}_{\perp}^{\prime}$, which is perpendicular to the $c$ axis and $\mathbf{k}_{\|}^{\prime}$, which is parallel to $c$. The proposed interlayer state ${ }^{10}$ of graphite can approximately be represented by Eqs. (10) and (11), as will be shown in Sec. IV. If Eqs. (10) and (11) apply, the squared TME of Eq. (5) equals the momentum-space density $\left|\left\langle p \mid \psi_{i}\right\rangle\right|^{2}$ of the initial state $\psi_{i}$ at $\mathbf{p} \equiv \mathbf{p}^{\prime}-\mathbf{q}$. Furthermore, let us assume that all final states, the momentum $p^{\prime}$ [Eq. (11)] of which meets

$$
\mathbf{p}^{\prime} \cdot \mathbf{q} \equiv p_{z}^{\prime}|\mathbf{q}|=\text { const },
$$

have the same energy transfer $\hbar \omega$. The corresponding mean-squared TME is then proportional to the Compton profile (CP)

$$
J\left(p_{z}\right) \equiv \int\left|\left\langle\mathbf{p} \mid \psi_{i}\right\rangle\right|^{2} d p_{x} d p_{y}
$$

with

$$
p_{z}=p_{z}^{\prime}-q \text {. }
$$

\section{B. Experiment, data processing}

The DDSCS was measured using synchrotron radiation from DORIS II by means of an experimental setup which is described in detail elsewhere. ${ }^{50-52}$ The graphite measurements were performed by using a Ge(311) crystal pair for monochromatization and a spherically bent $\mathrm{Si}(444)$ for analyzing of the scattered beam. The (variable) primary energy was around $8 \mathrm{keV}$. Within the useful bandwidth $2.3 \times 10^{10}$ photons $\mathrm{s}^{-1}$ could be supplied at the scattering sample, with the storage ring operated at $3.7 \mathrm{GeV}$ and $50 \mathrm{~mA}$. The $\mathrm{Ge}(311)$ monochromator and the $\mathrm{Si}(444)$ analyzer combined with the small angle $\left(\approx 1^{\circ}\right)$ between the crystal surfaces of the $\mathrm{Ge}(311)$ pair provide a nearly complete dispersion compensation. ${ }^{51}$ Thus a total-energy resolution of $0.8 \mathrm{eV}$ could be achieved, as could be concluded from the width of the quasielastically scattered line (Rayleigh line). The momentum transfer could be fixed to \pm 0.03 a.u., independently of the scattering angle $\theta$, that determines the momentum transfer $q$ via

$$
q=2 K_{0} \sin (\theta / 2) \text {. }
$$

Between $5 \times 10^{3}$ and $10^{4}$ counts were collected at the peak position of the inelastically scattered spectrum.

The sample of highly oriented pyrolytic (HOP) graphite (Union Carbide) was monochromator grade with less than 5 minutes of arc spread of the $c$ axis. The sample thickness was $1.3 \mathrm{~mm}$. The q\|c spectra were taken in reflection mode, the $q \perp c$ spectra in transmission mode. The experimental data were processed as follows:

(i) Subtraction of a linear background, found from the count rate on the high-energy side of the Rayleigh line.

(ii) Removing of the Rayleigh line, which was assumed to be symmetric and superimposed by the inelastically scattered part of the spectrum only on its low-energy side. Due to the relative high intensity of the Rayleigh line, its removal leads to less reliable inelastic spectra in the range of $0-3 \mathrm{eV}$ energy loss.

(iii) The inelastic spectra were brought to an absolute scale by utilizing the $f$ sum rule [Eq. (6)], where the $\omega$ integration should be over the whole valence-electron part of the spectrum. The normalization procedure can be justified in a strict sense only if the valence part of the spectrum is completely separated and independent of its core part. In reality there exists a small overlap of both parts on the energy scale. Therefore, the valence part was extrapolated into the range of the core contribution by means of an exponential, which was fitted to the low- 
energy tail of the valence spectrum. The error that could be introduced by an improper extrapolation was estimated to be less than $3 \%$. Moreover, also the valence part of the spectrum is influenced by the core polarizability via the contribution of the core to $\epsilon_{1}(q, \omega)$. If one approximates the core contribution to $\epsilon_{1}$ simply by the electronic part of the free-ion susceptibility of $\mathrm{C}^{4+}$, which is 0.0018 according to Ref. 53, it will be justified to neglect the valence-core interaction completely. The neglecting of the core contribution also applies to the KKT of the experimental spectra, where the valence electrons are treated as a closed system, independent of the core.

(iv) After transformation of the absolute valenceelectron $S(\mathbf{q}, \omega)$ data into $-\operatorname{Im}\left[\epsilon^{-1}(\mathbf{q}, \omega)\right]$ data [see Eq. (2)], the latter were subjected to the KKT according to Eqs. (7)-(9) by using a numerical method of Ref. 54.

\section{RESULTS AND GENERAL DISCUSSION}

\section{A. Core excitation}

The right-hand part of Fig. 1 presents the core contribution to the inelastic spectrum with $q=1.67 \mathrm{a} . \mathrm{u}$. for $\mathrm{q} \| \mathrm{c}$ and $q \perp c$, respectively, as obtained by subtracting the (extrapolated) part of the valence spectrum from the total spectrum. Considering the number of core electrons (two) and valence electrons (four), the scaling factor used for the core contribution was twice the scaling factor that was deduced by applying the $f$-sum rule to the valence part of the spectrum.

According to Eqs. (2)-(5), the core spectra should reflect almost exclusively the behavior of $\epsilon_{2}(q, \omega)$, since in this region of energy transfer $\epsilon_{1}(q, \omega) \approx 1$ and $\epsilon_{2}(\mathrm{q}, \omega) \ll<1$

Because the initial state (core state) of the relevant transition does not exhibit considerable $\mathbf{k}$ dispersion, $\epsilon_{2}(q, \omega)$ is determined by the density of the final states via the $\delta$ function in Eq. (5). The DOS is weighted by the

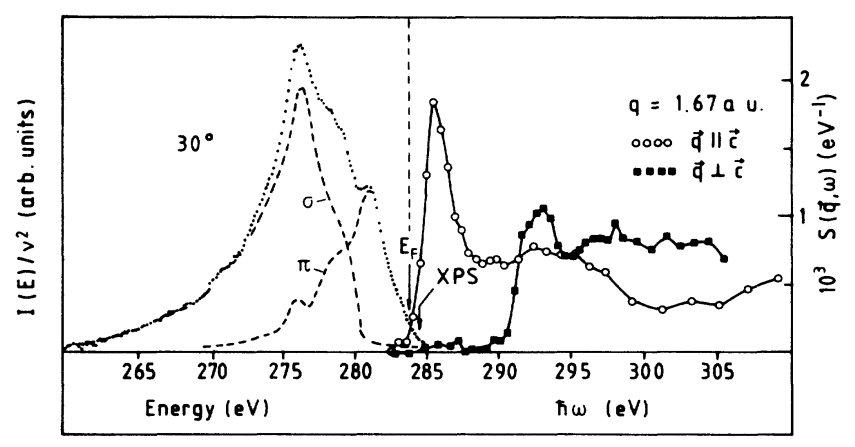

FIG. 1. Right-hand part, right-hand scale: core contribution to $S(\mathrm{q}, \omega)$ of HOP graphite for $\mathbf{q} \| \mathbf{c}$ (open circles) and $\mathbf{q} \perp \mathbf{c}$ (squares). Left-hand part, left-hand scale: $\mathrm{C} K$-emission band of single-crystalline graphite of Ref. 19 , take-off angle $30^{\circ}$. The decomposition into $\pi$ and $\sigma$ contributions was done by using the results of various take-off angles. $E_{F}$ : estimated position of the Fermi level. XPS: $1 s$ binding energy according to XPS measurements of Ref. 11. squared TME. The dependence of the TME on the $q$ value is only weak, but the TME strongly selects the final states according to their symmetry. The symmetry selectivity can be well understood within the limits of the dipole approximation, where the scattering operator $\exp (i \mathrm{q} \cdot \mathbf{r})$ of the TME is replaced by $i \mathrm{q} \cdot \mathbf{r}$. The dipole approximation $^{55}$ is justified, if $q a<<1$ [ $a$ is the core radius; $a \simeq 0.17$ a.u. for C $1 s$ (Ref. 56)]. The dipole-allowed transitions from the $1 s$ core state for graphite (space group $D_{6 h}^{4}$ ) are summarized in Table I, following from Ref. 57. As long as states of a 2D band structure are concerned, for $q \| \mathrm{c}$ solely transitions to $\pi^{*}$ states and for $\mathrm{q} \perp \mathrm{c}$ solely transitions to $\sigma^{*}$ states are dipole allowed. Thus, within the limits of a 2D description of the graphite band structure, the $q \| c$ spectrum exclusively reflects the partial DOS of unoccupied $\pi^{*}$ states, whereas the $q \perp c$ spectrum gives information about the partial DOS of the unoccupied $\sigma^{*}$ states. The strong symmetry selectivity points at the advantage of IXSS over EELS insofar as the direction of $\mathbf{q}$ is independent of $\hbar \omega$ and $\mathbf{q}$ can be moved freely through the bulk specimen.

Transitions to states on symmetry points and symmetry lines with $k_{z} \neq 0$ are less symmetry selective as shown in Table I. However, for final states $\left|\mathbf{k}^{\prime}, l^{\prime}\right\rangle$ with weak $k_{z}$ dispersion the TME $\left\langle\mathbf{k}^{\prime}, l^{\prime}|\exp (i \mathrm{q} \cdot \mathbf{r})| 1 s\right\rangle$ in Eq. (5) can be well approximated by $\left\langle\mathbf{k}_{1}^{\prime}, l^{\prime}|\exp (i \mathrm{q} \cdot \mathbf{r})| 1 s\right\rangle$ due to the weak interlayer interaction, where $k_{1}^{\prime}$ is the component of $\mathbf{k}^{\prime}$ perpendicular to the $c$ axis. Therefore, in cases of weak $k_{z}$ dispersion, the selection rules valid for $\mathbf{k}_{\perp}^{\prime}$ can be approximately applied to states $\left|\mathbf{k}^{\prime}, l^{\prime}\right\rangle$ throughout the Brillouin zone.

The interpretation of the core-excitation spectra, as will be given in Sec. IV, strongly relies on the energy position of the Fermi level. This position was found by combining our core-excitation spectra with ARXES of Ref. 19 , brought roughly to the same scale as shown in Fig. 1. The energy of the Fermi level was then fixed in the usual heuristic manner at the intersection of both spectra, yielding $E_{F}=283.8 \pm 0.3 \mathrm{eV}$. This value has to be compared with XPS measurement of the $1 \mathrm{~s}$ binding energy $E_{B}$ of carbon in graphite, $E_{B}=284.4 \mathrm{eV}$, based on an excitation energy of $1486.6 \mathrm{eV}(\mathrm{Al} K \alpha){ }^{11}$ The difference between both values may partly be due to the

TABLE I. Dipole-allowed transitions from a $1 s$ core state into states of graphite (space group $D_{6 h}^{4}$ ). Notation according to Ref. 57.

\begin{tabular}{cc}
\hline \hline & Transitions from: $1 s$ core state \\
\hline To & $\Gamma_{2}^{-}, M_{4}^{-}, K_{4}, \Sigma_{3}, \Lambda_{3}, T_{3}$ \\
for q\|c & $A_{1}, H_{1}, L_{1}, \Delta_{1}, \Delta_{2}, U_{1}$ \\
& $U_{2}, P_{1}, P_{2}, S_{1}, R_{3}$ \\
To & $\Gamma_{6}^{-}, M_{2}^{-}, M_{3}^{-}, K_{5}, \Sigma_{1}, \Sigma_{4}$ \\
for q\c & $\Lambda_{1}, \Lambda_{4}, T_{1}, T_{4}$ \\
& $A_{3}, H_{2}, H_{3}, L_{1}, L_{2}, \Delta_{5}, \Delta_{6}$ \\
& $U_{1}, U_{2}, U_{3}, U_{4}, P_{3}, S_{1}, R_{1}, R_{4}$ \\
\hline
\end{tabular}


lower resolution of the IXSS spectra compared with ARXES but could also be attributed to core-hole relaxation. The threshold of the core-excitation spectrum occurs at the relaxed energy. Due to the low energy of the excited electron, the final states have time enough to relax, so that the core-hole potential can be considered as switched on adiabatically. On the contrary, in the photoemission process the ejected electron has more than 1 $\mathrm{keV}$ surplus energy, so that shakeup satellites build up a spectrum that lies above the adiabatic threshold energy. ${ }^{58,59}$

As already pointed out, the core-excitation spectra of graphite obtained so far by EELS (Refs. 28, 33, and 34) can be understood as a superposition of our $\mathbf{q} \perp \mathbf{c}$ and $\mathbf{q} \| \mathbf{c}$ spectra. The high q selectivity of IXSS can not be achieved by EELS for principle experimental reasons. Nevertheless, the energies of the main edges and peaks of the EELS data agree quite well with ours. The same is true with the SXAS data of graphite. ${ }^{31}$ Within the limits of the dipole approximation these spectra are dominated by a TME, in which the polarization vector $\hat{\mathbf{e}}$ of the $\mathrm{ab}$ sorbed radiation plays the same role as $q$ in IXSS. ${ }^{60}$ With SXAS it is mainly the difficulty connected with the preparation of appropriately oriented samples that makes the full utilization of the $\hat{\mathbf{e}}$ selectivity impossible. Again the energy of the main edges and peaks of the "superimposed" SXAS spectra are in accordance with ours.

\section{B. Interband transitions; $q$ parallel to the $c$ axis}

In Fig. 2(a) the inelastic spectra after subtraction of both the Rayleigh line and the background, and after normalization to absolute values of $S(\mathrm{q}, \omega)$, are shown for q parallel to the $c$ axis and $0.37<q<2.06$ a.u. In Fig. 2(b) the corresponding real and imaginary parts of $\epsilon(q, \omega)$ are plotted as obtained by Kramers-Kronig transformation according to Eqs. (7)-(9). The $S(q, \omega)$ spectra exhibit a sharp peak, the energetic position of which oscillates between 15 and $19 \mathrm{eV}$. The $\epsilon(\mathrm{q}, \omega)$ spectra of Fig. 2(b) prove that this peak originates in a corresponding peak of $\epsilon_{2}(q, \omega)$ which also has a weak oscillatory dispersion behavior. The peak was not detected by previous lowresolution IXSS measurements, ${ }^{45}$ possibly because of the strong overlap of the Rayleigh line.

EELS spectra with a large q component parallel to the $c$ axis $^{41,42}$ have revealed the peak for $q<0.2$ a.u., where again the pure $q \| c$ case could not be realized. Provided the peak is attributed to a high value of the JDOS for certain interband transitions, its weak dispersion can be understood as a consequence of the weak $k_{z}$ dispersion of the graphite 2D bands up to $20 \mathrm{eV}$ (and more) above the Fermi energy. Within the limits of a $2 \mathrm{D}$ description of the graphite band structure only vertical transitions have to be taken into account for $q$ parallel to the $c$ axis. Again, dipole selection rules may help us to select those interband transitions that can contribute to the $q \| \mathbf{c}$ spectra. Inspection of Table II shows that only $\sigma \rightarrow \pi^{*}$ and $\pi \rightarrow \sigma^{*}$ transitions are dipole allowed.

Between 3D bands the dipole selection rules are less exclusive for transitions with $q \| c$. But the same arguments in favor of a 2D approximation of the graphite band
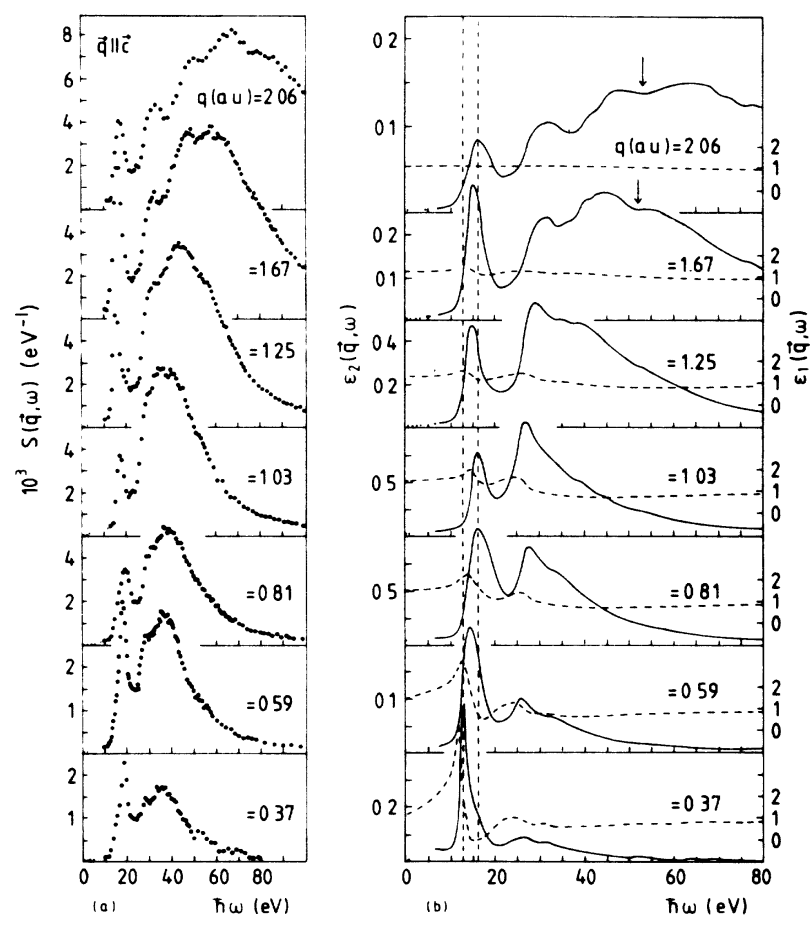

FIG. 2. (a) $S(q, \omega)$ of HOP graphite for $q$ parallel to the $c$ axis; $q$ values are given in the insets. Note the different ordinate scale. The small dots in the region of low-energy transfer $\omega$ represent that part of the spectra which is less reliable because of heavy superposition by the Rayleigh line in the rough data. (b) $\epsilon_{1}(\mathbf{q}, \omega)$ (dashed curve, right-hand scale) and $\epsilon_{2}(\mathbf{q}, \omega)$ (solid line, left-hand scale) of HOP graphite for $q$ parallel to the $c$ axis; $q$ values are given in the insets. Note the different ordinate scale. The straight dashed lines serve as a guide for the eye and connect the limiting positions of the first peak of $\epsilon_{2}(\mathbf{q}, \omega)$.

structure as given in Sec. III A also holds for interband transitions to a certain extent.

If one can vary the $q$ values within a large range, a further discrimination between possible interband transitions will be provided by the $q$ dependence of the TME's, as already discussed in Sec. II A.

A shoulder (peak) around $30 \mathrm{eV}$ is a further structure of the $S(\mathrm{q}, \omega)$ spectra of Fig. 2(a), which is nearly nondispersing and leads for $q \leq 1.03$ a.u. to a marked peak of $\epsilon_{2}$ with an energy position between 26 and $28 \mathrm{eV}$, together with a less pronounced shoulder between 32 and $34 \mathrm{eV}$. For $q \geq 1.25$ a.u., this shoulder seems to grow into a dominating peak at $33 \mathrm{eV}$.

Let us now consider the broad part of the $S(\mathrm{q}, \omega)$ spectrum as a whole, the center of gravity of which is strongly positive dispersive. The physical reason for the $q$ dependence of the center-of-gravity position is for $q \leq 1$ a.u. mainly the local minimum of $\epsilon_{1}(q, \omega)$. But for $q>1$ a.u. it is more and more the $q$ dependence of the TME's of Eq. (5) for transitions into continuum states that determines the shape of the $\epsilon_{2}(q, \omega)$ spectra, and, because of the nearly structureless behavior of $\epsilon_{1}$, also the shape of the 
TABLE II. Dipole-allowed vertical transitions in HOP graphite (space group $D_{6 h}^{4}$ ). Notation according to Ref. 57 . Only states that are relevant to a 2D description of the graphite band structure are included.

\begin{tabular}{|c|c|c|c|c|c|c|}
\hline Transitions from: & $\Gamma_{1}^{ \pm}$ & $\Gamma_{2}^{ \pm}$ & $\Gamma_{\frac{ \pm}{3}}^{ \pm}$ & $\Gamma_{4}^{ \pm}$ & $\Gamma_{\frac{1}{5}}^{ \pm}$ & $\Gamma_{6}^{ \pm}$ \\
\hline $\begin{array}{l}\text { To } \\
\quad \text { for } \mathbf{q} \| \mathbf{c}\end{array}$ & $\Gamma_{2}^{\mp}$ & $\Gamma_{1}^{\mp}$ & $\Gamma_{4}^{\mp}$ & $\Gamma_{3}^{\mp}$ & $\Gamma_{s}^{\mp}$ & $\Gamma_{6}^{\mp}$ \\
\hline $\begin{array}{l}\text { To } \\
\quad \text { for } q \perp c\end{array}$ & $\Gamma_{6}^{\mp}$ & $\Gamma_{6}^{\mp}$ & $\Gamma_{5}^{\mp}$ & $\Gamma_{5}^{\mp}$ & $\Gamma_{3}^{\mp}, \Gamma_{4}^{\mp}, \Gamma_{6}^{\mp}$ & $\Gamma_{1}^{\mp}, \Gamma_{2}^{\mp}, \Gamma_{5}^{\mp}$ \\
\hline Transitions from: & \multicolumn{2}{|c|}{$M_{1}^{ \pm}$} & \multicolumn{2}{|c|}{$M_{2}^{ \pm}$} & $M_{3}^{ \pm}$ & $M_{4}^{ \pm}$ \\
\hline $\begin{array}{l}\text { To } \\
\quad \text { for } \mathbf{q} \| \mathbf{c}\end{array}$ & \multicolumn{2}{|c|}{$M_{4}^{\mp}$} & & $M_{2}^{\mp}$ & $M_{1}^{\mp}$ \\
\hline $\begin{array}{l}\text { To } \\
\quad \text { for } q \perp c\end{array}$ & \multicolumn{2}{|c|}{$M_{3}^{\mp}, M_{2}^{\mp}$} & \multicolumn{2}{|c|}{$M_{1}^{\mp}, M_{4}^{\mp}$} & $M_{1}^{\mp}, M_{4}^{\mp}$ & $M_{2}^{\mp}, M_{3}^{\mp}$ \\
\hline Transitions from: & $K_{1}$ & $K_{2}$ & $K_{3}$ & $K_{4}$ & $K_{5}$ & $K_{6}$ \\
\hline $\begin{array}{l}\text { To } \\
\quad \text { for } \mathbf{q} \| \mathbf{c}\end{array}$ & $K_{4}$ & $K_{3}$ & $K_{2}$ & $K_{1}$ & $K_{6}$ & $K_{5}$ \\
\hline $\begin{array}{l}\text { To } \\
\text { for } q \perp c\end{array}$ & $K_{5}$ & $K_{6}$ & $K_{5}$ & $K_{6}$ & $K_{1}, K_{3}, K_{5}$ & $K_{2}, K_{4}, K_{6}$ \\
\hline Transitions from: & \multicolumn{2}{|c|}{$\Sigma_{1}$} & \multicolumn{2}{|c|}{$\Sigma_{2}$} & $\Sigma_{3}$ & $\Sigma_{4}$ \\
\hline $\begin{array}{l}\text { To } \\
\quad \text { for } q \perp \mathbf{c}\end{array}$ & \multicolumn{2}{|c|}{$\Sigma_{3}$} & \multicolumn{2}{|c|}{$\Sigma_{4}$} & $\Sigma_{1}$ & $\Sigma_{2}$ \\
\hline $\begin{array}{l}\text { To } \\
\text { for } q \perp c\end{array}$ & \multicolumn{2}{|c|}{$\Sigma_{1}, \Sigma_{4}$} & \multicolumn{2}{|c|}{$\Sigma_{2}, \Sigma_{3}$} & $\Sigma_{2}, \Sigma_{3}$ & $\Sigma_{1}, \Sigma_{4}$ \\
\hline Transitions from: & \multicolumn{2}{|c|}{$T_{1}$} & \multicolumn{2}{|c|}{$T_{2}$} & $T_{3}$ & $T_{4}$ \\
\hline $\begin{array}{l}\text { To } \\
\quad \text { for } \mathbf{q} \| \mathbf{c}\end{array}$ & \multicolumn{2}{|c|}{$T_{3}$} & \multicolumn{2}{|c|}{$T_{4}$} & $T_{1}$ & $T_{2}$ \\
\hline $\begin{array}{l}\text { To } \\
\text { for } q \perp \mathbf{c}\end{array}$ & \multicolumn{2}{|c|}{$T_{1}, T_{4}$} & \multicolumn{2}{|c|}{$T_{2}, T_{3}$} & $T_{2}, T_{3}$ & $T_{1}, T_{4}$ \\
\hline Transitions from: & \multicolumn{2}{|c|}{$\Lambda_{1}$} & \multicolumn{2}{|c|}{$\Lambda_{2}$} & $\Lambda_{3}$ & $\Lambda_{4}$ \\
\hline $\begin{array}{l}\text { To } \\
\quad \text { for } q \| c\end{array}$ & \multicolumn{2}{|c|}{$\Lambda_{3}$} & \multicolumn{2}{|c|}{$\Lambda_{4}$} & $\Lambda_{1}$ & $\Lambda_{2}$ \\
\hline $\begin{array}{l}\text { To } \\
\text { for } q \perp c\end{array}$ & \multicolumn{2}{|c|}{$\Lambda_{1}, \Lambda_{4}$} & \multicolumn{2}{|c|}{$\Lambda_{2}, \Lambda_{3}$} & $\Lambda_{2}, \Lambda_{3}$ & $\Lambda_{1}, \Lambda_{4}$ \\
\hline
\end{tabular}

$S(\mathbf{q}, \omega)$ spectra. Since the continuum states can be considered as nearly-free-electron-like, the $q$ dependence of the TME's applies as discussed in Sec. II A [Eqs. (10)-(14)]. Thus with increasing $q$ the $S(\mathbf{q}, \omega)$ spectra continuously change over into Compton profiles. It is worth noting that there remains a nondispersive structure in $\epsilon_{2}$, in addition to that already discussed, mainly a weak dip around $53 \mathrm{eV}$ which clearly evolves for $q \geq 1.67$ a.u. [arrow in Fig. 2(b)]. This dip will be discussed in connection with a similar but much stronger structure in the q $\perp$ c spectra.

\section{Interband transitions, $q$ perpendicular to the $c$ axis, $q$ in variable directions}

In Fig. 3(a) the inelastic spectra after normalization to absolute values of $S(\mathbf{q}, \omega)$ are shown for $\mathbf{q} \perp \mathbf{c}$ and $0.37<q<2.06$ a.u. In Fig. 3(b) the corresponding real and imaginary parts of $\epsilon(\mathbf{q}, \omega)$ are plotted. The $S(\mathbf{q}, \omega)$ spectra for the smallest value of $q$ exhibit peaks (shoulders) around 10,16,30, and $48 \mathrm{eV}$, that show more or less positive dispersion, with some structures fading out. The spectra resemble for $q \leq 0.59$ a.u. quite well the $q$ - 

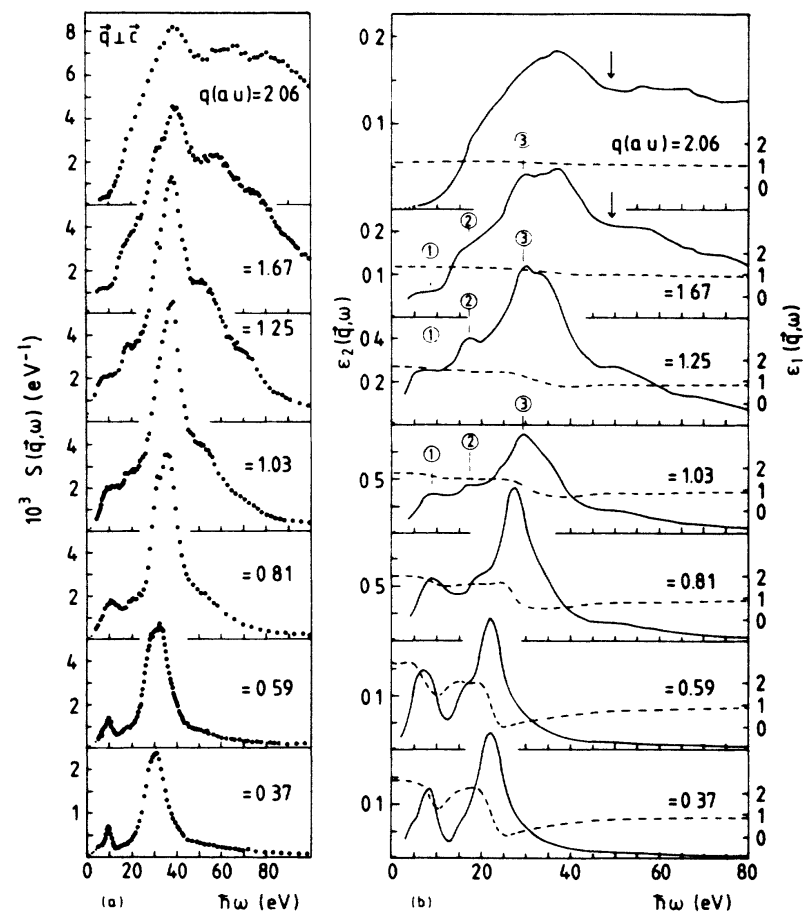

FIG. 3. (a) $S(\mathbf{q}, \omega)$ of HOP graphite for q perpendicular to the $c$ axis; $q$ values are given in the insets. Note the different ordinate scales. Small dots mark regions of less reliability. (b) $\epsilon_{1}(\mathbf{q}, \omega)$ (dashed curve, right-hand scale) and $\epsilon_{2}(\mathbf{q}, \omega)$ (solid line, left-hand scale) of HOP graphite for $q$ perpendicular to the $c$ axis; $q$ values are given in the insets. Note the different ordinate scales. Numbered marks set off peaks or shoulders of the fully developed $\epsilon_{2}$ fine structure.

dependent EELS spectra of equivalent $q$ orientation of Ref. 44 as far as the energy region $\hbar \omega<40 \mathrm{eV}$ is concerned, where multiple scattering is not that important.

For large values of $q(q \geq 1.0$ a.u. $)$ the $\epsilon_{2}$ spectra exhibit a fine structure, which shows negligible dispersion [see the numbered marks in Fig. 3(b)].

Within the limits of the 2D band structure, the dipole selection rules allow exclusively $\sigma \rightarrow \sigma^{*}$ and $\pi \rightarrow \pi^{*}$ transitions for $q \perp c$. The corresponding vertical transitions are listed in Table II. For $q \perp c$ finite values of $q$ lead to nonvertical transitions in the 2D band structure. The development with increasing $q$ of a nearly nondispersive fine structure of $\epsilon_{2}$ can be understood as a consequence of nonvertical transitions on the one hand and the rotational disorder of HOP graphite on the other. Provided $q$ is larger than half the Brillouin-zone diameter, the rotational disorder generates a much larger variety of nonvertical transitions for a given value of $q$ than in the case of single crystals. As a result, the same nonvertical transition connected with a high JDOS may occur at quite different values of $q$. Thus a nearly- $q$-independent fine structure of $\epsilon_{2}$ becomes plausible. However, the unambiguous attribution of peaks in $\epsilon_{2}$ to certain nonvertical transitions seems to be very difficult, so that we have not tried to compare the qlc spectra with band-structure calcula- tions.

For $q$ values larger than 1.67 a.u., the $q$-independent fine structure is fading out, so all that remains is a dip in $\epsilon_{2}$ around $49 \mathrm{eV}$ [arrows in Fig. 3(b)] which in Ref. 45 also was seen for $q \perp c$ and was interpreted to be an indication for the universal double-peak structure in the dynamic structure factor $S(\mathbf{q}, \omega)$ of a homogeneous electron liquid, where this fine structure has been attributed to short-range electron-electron correlations. ${ }^{61}$ The authors of Ref. 45 felt their view supported by the absence of a similar structure in the $q \| \mathbf{c}$ spectra. Anomalies of the measured static structure factor of $B e$ and graphite $(q \perp c)$ (Ref. 62) can also be understood as indications of special features in the electron-electron correlation at short distances. A quite different interpretation, as already given in connection with our $\mathrm{Li}$ and $\mathrm{Be}$ measurements, ${ }^{52,63}$ cannot be excluded because of both the occurrence of a similar, even though weaker, dip in our q\|l spectra and the q-orientation dependence of the dip position: These dips could also be due to excitation gaps for transitions ending up on Bragg planes in the extended-zone scheme.

Finally, Fig. 4(a) shows the inelastic spectra of HOP graphite after normalization to absolute values of $S(q, \omega)$ for $q=0.59$ a.u., with $\alpha$ the deviation of $q$ from the $c$ axis. In Fig. 4(b) the corresponding real and imaginary parts of $\epsilon(\mathbf{q}, \omega)$ are plotted. Within the limits of the dipole approximation, according to Eq. (5), the $\epsilon_{2}(q, \omega)$ spectra of
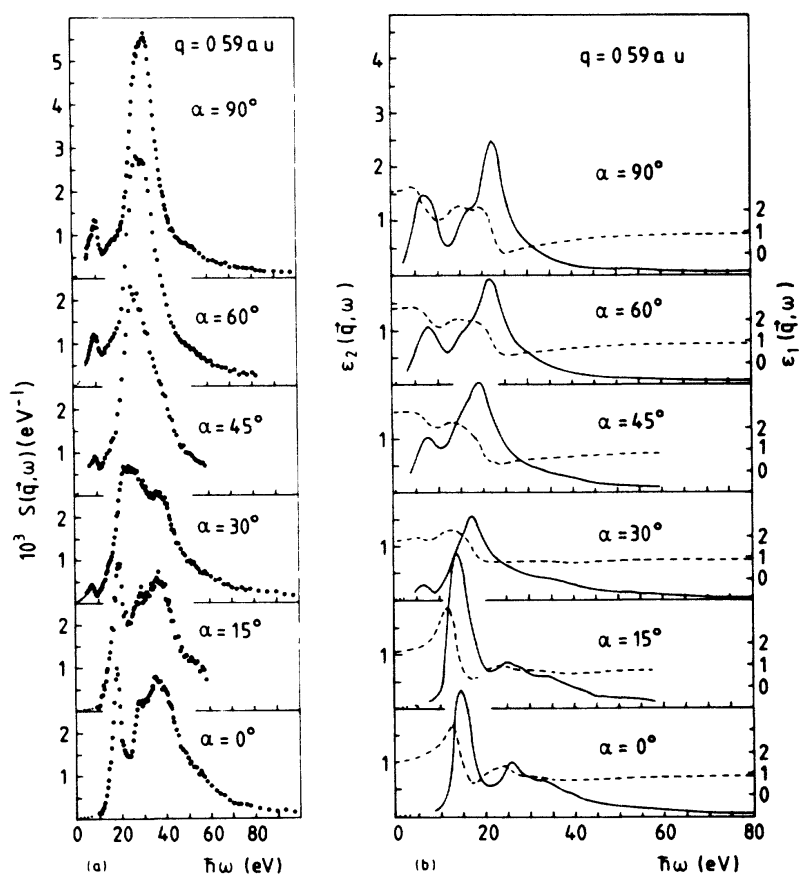

FIG. 4. (a) $S(\mathrm{q}, \omega)$ of HOP graphite for $q=0.59$ a.u. The direction of $\mathbf{q}$ makes an angle $\alpha$ with the $c$ axis. (b) $\epsilon_{1}(\mathbf{q}, \omega)$ (dashed curve, right-hand scale) and $\epsilon_{2}(\mathbf{q}, \omega)$ (solid line, lefthand scale) of HOP graphite for $q=0.59$ a.u. The direction of $q$ makes an angle $\alpha$ with the $c$ axis. 
Fig. 4(b) can be understood as a weighted superposition of a $q \| c$ spectrum and a $q \perp c$ spectrum with weights $\cos ^{2} \alpha$ $\left(\sin ^{2} \alpha\right)$ for $q_{\|}=q \cos \alpha\left(q_{\perp}=q \sin \alpha\right)$, respectively. At the same time, the $S(\mathrm{q}, \omega)$ spectra obviously cannot be understood as a simple linear superposition of the relevant q\|c spectra with qLc spectra because of the nonlinear relationship between $\epsilon_{1}(q, \omega), \epsilon_{2}(q, \omega)$, and $\operatorname{Im}\left[\epsilon^{-1}(q, \omega)\right]$ [see Eqs. (7)-(9)].

\section{COMPARISON WITH BAND-STRUCTURE CALCULATION AND OTHER SPECTROSCOPIES}

\section{A. Core-excitation spectra}

As discussed in Sec. III A, the q\|c core-excitation spectrum (see Fig. 1) reflects the density of unoccupied $\pi$ states of a $2 \mathrm{D}$ band structure weighted by the TME of Eq. (5). The most prominent structure of this spectrum is the strong peak $1.6 \pm 0.3 \mathrm{eV}$ above the Fermi energy $E_{F}$, which we will interpret as due to the high density of unoccupied $\pi$ states near the $M$ point. According to the dipole selection rules, the energy of this peak can be identified with the energy position of the $M_{4}^{-}$state above $E_{F}$, which is $1.4,1.7$, and $1.5 \mathrm{eV}$ in Refs. 22, 8, and 9, respectively (see Fig. 5 from Ref. 8). Thus the experimental value does not fall far from the available theoretical ones. Nondispersive structures in ARIPS at $\simeq 2 \mathrm{eV}$ above $E_{F}$ in Ref. 25 and at $1.5 \mathrm{eV}$ in Ref. 26 can also be ascribed to a high DOS, if they are interpreted as due to non- $k_{\|}$conserving emission. Our interpretation of the $1.6-\mathrm{eV}$ peak gets further support by the well-known peak at 4.5 $\mathrm{eV}$ in $\epsilon_{2}(q=0, \omega)$ spectra of graphite as deduced both from optical measurements ${ }^{40}$ and EELS. ${ }^{42}$ This peak was attributed to a $M_{3}^{+} \rightarrow M_{4}^{-}$transition. Taking into account the experimentally well-established $M_{3}^{+}$position, which is observed ${ }^{15-18}$ universally at $\simeq 3 \mathrm{eV}$ below $E_{F}$, one again obtains $1.5 \mathrm{eV}$ above $E_{F}$ for the $M_{4}^{-}$position. A similar peak at a core-excitation energy of $285.4 \mathrm{eV}$ has also been identified in EELS (Refs. 33 and 34) and SXAS (Ref. 31). In Ref. 34 the peak is interpreted as due to a core exciton. In view of all the findings mentioned above, the exciton interpretation seems not to be conclusive. Finally, the deconvoluted SXAPS (Ref. 35) indicates a strong peak of the DOS just $1.7 \mathrm{eV}$ above $E_{F}$.

Further structure in the $\mathbf{q} \| \mathbf{c}$ core-excitation spectrum is less distinct and cannot be attributed with some confidence to features of the band structure.

The qlc core-excitation spectrum (see Fig. 1) which, according to Sec. III A, reflects transitions into $\sigma$ states of a 2D band structure of graphite, is effectively free of any transitions to $6.4 \mathrm{eV}$ above $E_{F}$. Then a steep rise begins with an inflection point at $7.3 \pm 0.3 \mathrm{eV}$, followed by a somewhat asymmetric peak at $9.0 \pm 0.3 \mathrm{eV}$. A DOS peak at this position has already been found in deconvoluted SXAPS, ${ }^{35}$ EELS, $^{33}$ and SXAS. ${ }^{31}$ However, these methods were not symmetry selective enough to attribute this peak unambiguously to a high DOS of exclusively $\sigma^{*}$ states. Considering recent band-structure calculations $^{22,8,9}$ (the results of Ref. 8 are plotted in Fig. 5 and should serve as a guide) combined with the dipole selection rules (Table $I$ ), it seems reasonable to associate the
9-eV peak with the high DOS both near $M_{2}^{-}$and on $\Sigma_{1}$ between $\Gamma_{6}^{-}$and $M_{2}^{-}$. The calculated energy bands ${ }^{22,8,9}$ between $\Gamma_{6}^{-}$and $M_{2}^{-}$cover the following ranges (energy values above $E_{F}$ ): $8.0-7.5 \mathrm{eV}$ in Ref. $22 ; 7.4-7.4 \mathrm{eV}$ in Ref. 8 and 9.2-7.6 eV in Ref. 9. Thus the centers of the calculated $\Gamma_{6}^{-}-M_{2}^{-}$bands and especially the position of $M_{2}^{-}$lie at smaller energies above $E_{F}$ than found in our spectra.

A similar discrepancy from calculations was also found in ARIPS of Refs. 25 and 26, in which $\Gamma_{6}^{-}, \Gamma_{5}^{+}$is at 9.5 and $9.8 \mathrm{eV}$ above $E_{F}$, respectively, and in which highDOS features near $M$ could be established at $8.5 \mathrm{eV}$ in either measurements in fairly good agreement with our spectra. If features at $7.5 \mathrm{eV}$ above $E_{F}$ as found in the secondary-electron emission of graphite ${ }^{15,18}$ are left unexplained, since no indication for similar structures could be seen in ARIPS, ${ }^{24,25,26}$ the calculation of Ref. 9 is nearest to the experiment in the case of the $\Gamma_{6}^{-}-M_{2}^{-}$

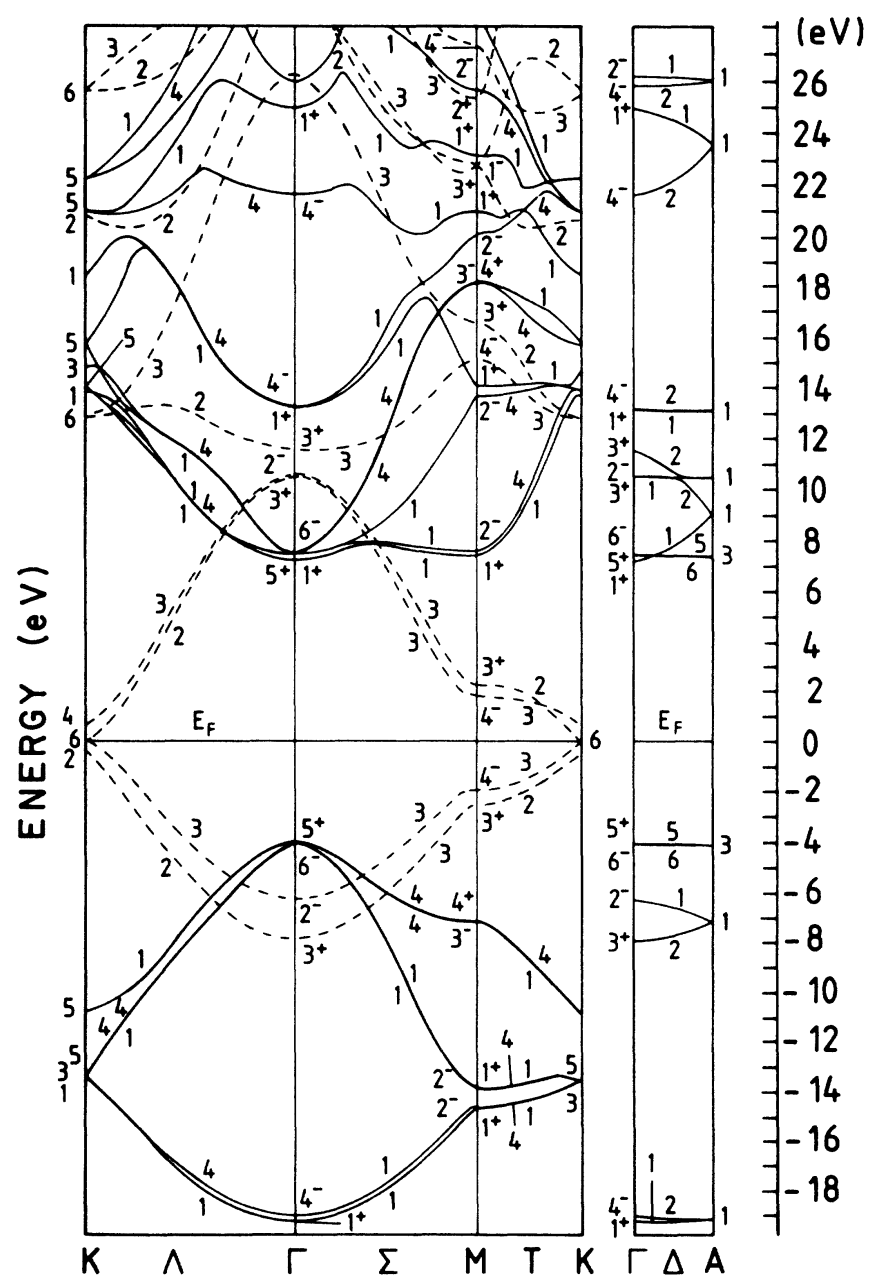

FIG. 5. Calculated band structure of graphite from Ref. 8 . Solid lines represent the $\sigma$ bands and the dashed lines represent the $\pi$ bands. 
band. The calculation of Ref. 9 has also located the socalled interlayer state with its strong $k_{\|}$dispersion nearest to all pertinent experiments. ${ }^{24,26}$ The interlayer state has $\Gamma_{1}^{+}$symmetry at the bottom of the band $17.0 \mathrm{eV}$ above $E_{F}$ in Ref. 8, see Fig. 5) and, furthermore, $\Delta_{1}$ and $A_{1}$ symmetry as far as its $k_{\|}$dispersion is concerned. Therefore, the above states of the interlayer band are not detectable in our $q \perp c$ core-excitation spectra for symmetry reasons (Table I). Even near the $\Gamma$ point, on $\Sigma_{1}$ and $\Lambda_{1}$, where a small $p$ admixture makes possible transitions from the $1 s$ core, this interlayer state will contribute to the $q \perp c$ core-excitation spectra to a negligible extent for two reasons. First of all, the strong $k_{\|}$dispersion will smear out the contributions. Secondly, the small overlap of the $1 s$ core with the interlayer region, where the interlayer state is concentrated, ${ }^{10}$ will hold down its contribution. Only when the interlayer band is approaching the zone boundary do both an increasing DOS and a decreasing $k_{\|}$dispersion cause the contribution of this band to the $q \perp c$ core-excitation spectra to increase. Therefore it seems reasonable, although not compelling, to interpret the steep rise of our spectrum at $7.3 \mathrm{eV}$ as an indication of the onset of the interlayer state contribution.

\section{B. Interband transitions}

As has been discussed in Sec. III B, the most prominent peaks of the $\epsilon_{2}(\mathbf{q}, \omega)$ spectra with $\mathbf{q} \| \mathbf{c}$, one around $15 \mathrm{eV}$, the other around $27 \mathrm{eV}$, have to be attributed ei- ther to $\sigma-\pi^{*}$ or to $\pi-\sigma^{*}$ high-JDOS vertical transitions of the $2 \mathrm{D}$ band structure. In addition, the transitions have to obey the selection rules as summarized in Table II. In order to find out relevant transitions in the calculated band structure, one has to look for corresponding nearly-parallel-band situations in plots of the $k_{x, y}$ dispersion. Taking the band structure of Ref. 8 as an example, nearly-parallel-band transitions are shown in Fig. 6(a) which can be made responsible for the " $15-e V$ peak."

Two nearly-parallel-band transitions are competing. The first one, marked by solid arrows in Fig. 6(a), is a transition between $\pi$ bands and the $\sigma$-type interlayer band, ${ }^{12}$ which exhibits a strong $k_{z}$ dispersion along $\Gamma A$. The second one, marked by dashed arrows in Fig. 6(a), is a $\sigma-\pi^{*}$ transition. The oscillating $q$ dependence of the $15-\mathrm{eV}$ peak suggests that the center of the transition energy for these two transitions is slightly different. The resulting observed peak positions would then depend on the relative $q$-dependent weight of each transition. In order to find out the center of gravity for the energy of the $\pi \rightarrow$ interlayer state transition, we have plotted in Fig. 7 its relative weight as a function of $q$ together with the $q$ dependence of the $15-\mathrm{eV}$ peak position. The relative weight was set equal to the $\pi$-Compton profile, $J\left(p_{z}\right)$, of graphite, ${ }^{64}$ where, according to Eqs. (10) - (14),

$$
p_{z}=p_{z}^{\prime}-q, \quad p_{z}^{\prime}=\left[2 m\left(E_{A_{1}}-E_{F}\right)\right]^{1 / 2} / \hbar
$$

and $E_{A_{1}}-E_{F}$, the energy above $E_{F}$ of the interlayer state

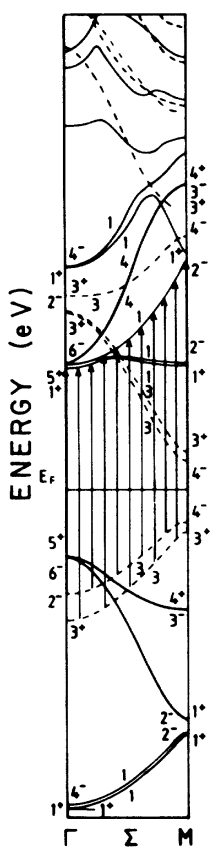

(a)
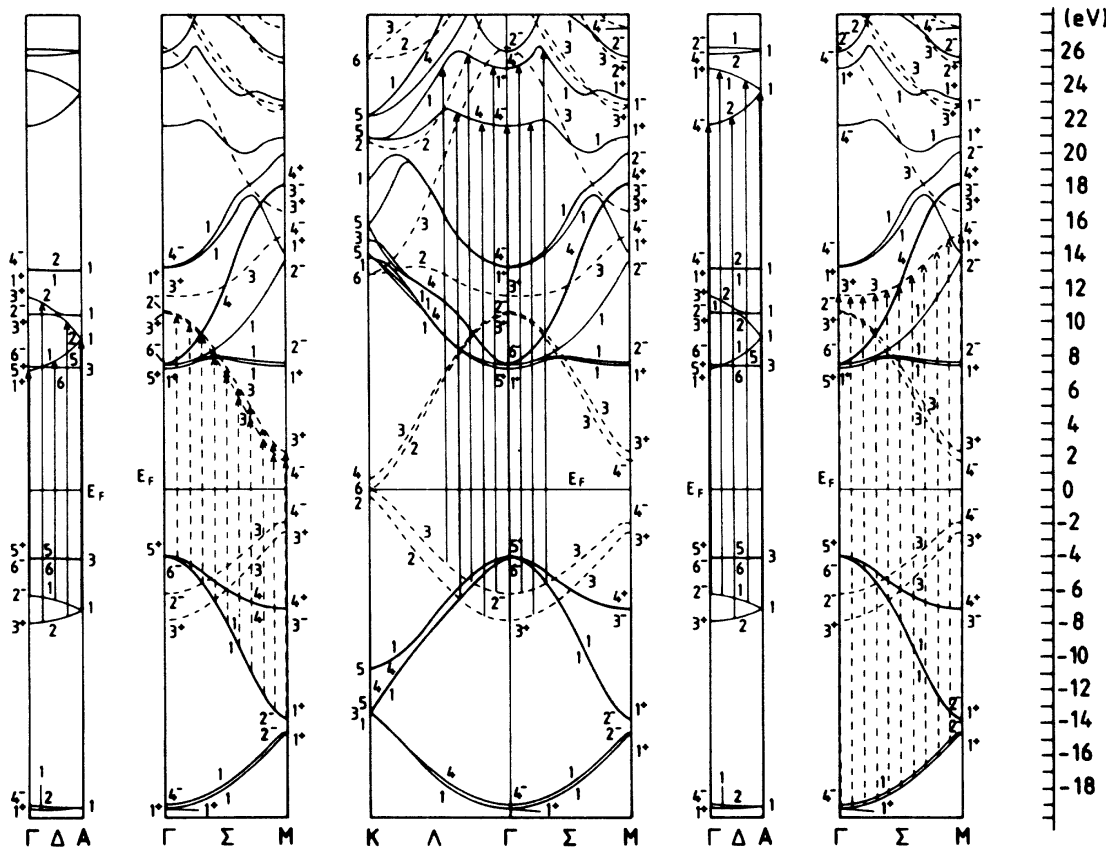

(b)

FIG. 6. Nearly-parallel-band transitions, dipole allowed for $\mathbf{q} \| \mathbf{c}$, in the graphite band structure of Ref. 8 . Solid lines represent the $\sigma$ bands and the dashed lines represent the $\pi$ bands. (a) Transitions with energies around $15 \mathrm{eV}$. Solid arrows represent the $\pi \rightarrow \sigma^{*}$ transition and dashed arrows represent the $\sigma \rightarrow \pi^{*}$ transition. (b) Transitions with energies around 27 and $32 \mathrm{eV}$. Solid arrows represent the $\pi \rightarrow \sigma^{*}$ transition and dashed arrows represent the $\sigma \rightarrow \pi^{*}$ transition. 


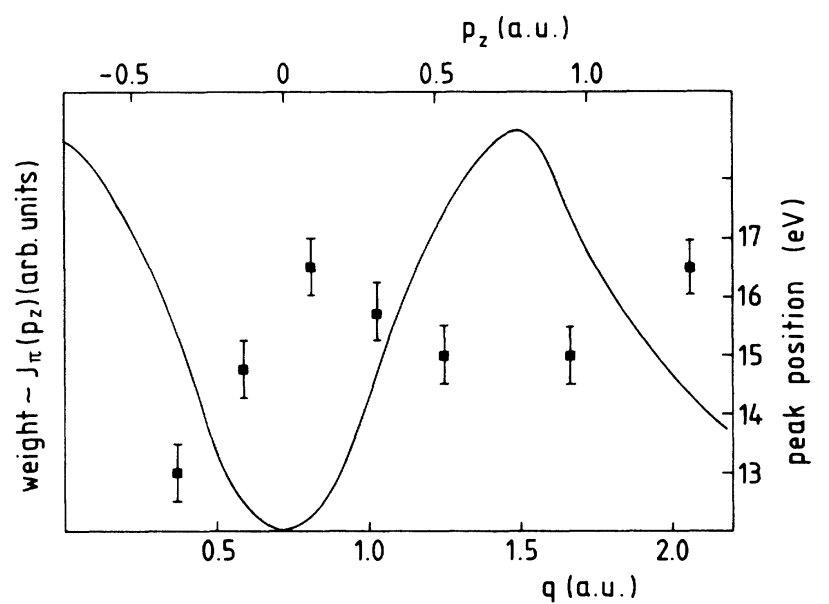

FIG. 7. The solid line is the relative weight of the $\pi \rightarrow$ interlayer state transition as a function of $q$ ( $\pi$-electron Compton profiles of Ref. 64); left-hand scale, arbitrary units. Squares represent the energy position of the $15-\mathrm{eV}$ peak of $\epsilon_{2}(\mathbf{q}, \omega)$ as a function of $q$, right-hand scale.

at $A_{1}$, was set at $7 \mathrm{eV}$, according to Ref. 9. $E_{A_{1}}$ in Eq. (15) was chosen to serve as a representative energy of the interlayer state, since, according to Ref. 9, for states near the $A_{1}$ point, the corresponding $E\left(k_{z}^{\prime}\right)$ follows approximately the free-electron-like relation

$$
\frac{\partial E\left(k_{z}^{\prime}\right)}{\partial k_{z}^{\prime}}=\hbar\left\{2\left[E\left(k_{z}^{\prime}\right)-E_{F}\right] / m\right\}^{1 / 2}
$$

Additionally, both the parallel-band situation, as assumed for these transitions with respect to the $k_{x, y}$ dispersion, and the $c$-axis orientation of $q$ automatically make $\hbar \omega$ the same for all final states with $\mathbf{p}^{\prime} \cdot \mathbf{q}=$ const [ $\mathbf{p}^{\prime}$, according to Eq. (11)]. Therefore, the estimation of the relative weight on the base of Eqs. (10)-(14) is justified, at least for the neighborhood of the $A$ point.

According to Fig. 7, a high weight of the transition to the interlayer state correlates with the low-energy position of the $15-\mathrm{eV}$ peak. Thus one can fix the center of gravity for the energy of this transition to be $14.0 \pm 0.5$ $\mathrm{eV}$. This value should be compared with the energy of both the $\Gamma_{2}^{-} \rightarrow \Gamma_{1}^{+}$and the $M_{3}^{+} \rightarrow M_{2}^{-}$transitions of various band-structure calculations as done in Table III. In Table III the calculated values for the $\Gamma$-point transition always have to be taken for the lower limit of the transition energy in question, since the $k_{z}$ dispersion has not been taken into account. Taking the photoemission results for the dispersion of the valence $\pi$ bands to be correct $\left[E_{B}=8.5 \mathrm{eV}\right.$ at $\Gamma$ and $E_{B}=3 \mathrm{eV}$ at $M$ (Refs. 15 and 18)] and assuming a simple parallel-band situation for the $\pi \rightarrow \sigma^{*}$ transition, we deduce the following upper
TABLE III. Transition energies in $\mathrm{eV}$ for $\pi \rightarrow$ interlayer state transitions of various band-structure calculations and of this experiment.

\begin{tabular}{lcccc}
\hline Transition & Ref. 22 & Ref. 8 & Ref. 9 & $\begin{array}{c}\text { This } \\
\text { experiment }\end{array}$ \\
\hline$\Gamma_{2}^{-} \rightarrow \Gamma_{1}^{+}$ & 14.3 & 13.6 & 10.6 & \\
$M_{3}^{+} \rightarrow M_{2}^{-}$ & 16.5 & 16.1 & 9.9 & $14.0 \pm 0.5$ \\
\hline \hline
\end{tabular}

limits for the energy position above $E_{F}$ of the interlayer state high-symmetry points (these are upper limits, because the $k_{z}$ dispersion has not been taken into account):

$$
E_{\Gamma_{1}^{+}}=5.5 \mathrm{eV}, E_{M_{2}^{-}}=11.0 \mathrm{eV} \text {. }
$$

If one takes these values for the upper limit, they are in reasonable agreement both with experimentally estimated values for the bottom of the band (above $E_{F}$ ) [ $E_{\Gamma_{1}^{+}}=4.0 \pm 0.5 \mathrm{eV}$ (Ref. 24), $E_{\Gamma_{1}^{+}}=5.0 \mathrm{eV}$ (Ref. 26)] and with our own core-excitation measurements (onset of interlayer state contribution, $7.3 \mathrm{eV} ; E_{M_{2}^{-}}=9.5 \pm 0.5 \mathrm{eV}$ ).

Among the band-structure calculations, those of Refs. 22 and 8 place the interlayer band at a considerable higher position, namely $E_{\Gamma_{1}^{+}}=7.6 \mathrm{eV}, E_{M_{2}^{-}}=14.0 \mathrm{eV}$ and $E_{\Gamma_{1}^{+}}=7.4 \mathrm{eV}, E_{M_{2}^{-}}=13.6 \mathrm{eV}$, respectively. Reference 9 has calculated a somewhat lower position for the interlayer band compared with our experimental results, namely $E_{\Gamma_{1}^{+}}=3.6 \mathrm{eV}, E_{M_{2}^{-}}=6.8 \mathrm{eV}$. Considering the experiment as an upper limit, the calculated $E_{\Gamma_{1}^{+}}$value of Ref. 9 seems to be in better agreement with the experiment than those of Ref. 22 and Ref. 8.

We find with Fig. 7 that the center of gravity of the $\sigma \rightarrow \pi^{*}$ transition energy can be fixed to be $16.5 \pm 0.5 \mathrm{eV}$. This value should be compared with the energy of the $M_{2}^{-} \rightarrow M_{3}^{+}, M_{1}^{+} \rightarrow M_{4}^{-}$transition, which is assumed to dominate all other transitions marked in Fig. 6(a) because of the high DOS associated with the $M$ point. The comparison as shown in Table IV illustrates the good agreement between experiment and the various calculations.

Similarly, relying again on the photoemission result for the $\sigma$-valence-band $M$-point position $\left[E_{B}=14.5 \pm 0.5 \mathrm{eV}\right.$

TABLE IV. Transition energies in $\mathrm{eV}$ for $\sigma \rightarrow \pi^{*}$ transitions at the $M$ point of various band-structure calculations and of this experiment.

\begin{tabular}{ccccc}
\hline \hline Transition & Ref. 22 & Ref. 8 & Ref. 9 & $\begin{array}{c}\text { This } \\
\text { experiment }\end{array}$ \\
\hline$M_{2}^{-} \rightarrow M_{3}^{+}$ & 16.9 & 16.5 & 15.5 & \\
$M_{1}^{+} \rightarrow M_{4}^{-}$ & 16.3 & 16.1 & 16.4 & $16.5 \pm 0.5$ \\
\hline \hline
\end{tabular}


(Refs. 13 and 18)], we deduce $E_{M_{3}^{+}, M_{4}^{-}}=2.0 \pm 0.5 \mathrm{eV}$, which agrees well with our core-excitation results and the results of Refs. 25 and 26.

In Fig. 6(b) two different nearly-parallel-band transitions can be made responsible for the " $27-\mathrm{eV}$-peak" of $\epsilon_{2}(q, \omega)$ and for the shoulder at $32 \mathrm{eV}$, that becomes dominant for $q \gg 1.67$ a.u. The first transition is $\pi \rightarrow \sigma^{*}$ (solid arrows) and has parallel-band character only close to $\Gamma$, where the final $\sigma$ state exhibits a strong $k_{z}$ dispersion. Therefore, the same arguments for the $q$ dependence of the TME as in the interlayer state are applicable. Using $E_{A_{1}}-E_{F} \simeq 23 \mathrm{eV}$ in Eq. (16), we find a high contribution to $\epsilon_{2}$ of the $\pi \rightarrow \sigma^{*}$ transitions for $0.2<q<1.0$ a.u., which is precisely that $q$ region where the $27-\mathrm{eV}$ peak is dominating. Thus we attribute the 27 eV peak to the $\pi \rightarrow \sigma^{*}$ transition. Based on the photoemission-deduced binding energy of the $\pi$-valencestate $\Gamma$ point, $E_{B}=8.5 \mathrm{eV},{ }^{13,18}$ we obtain $18.5 \mathrm{eV}$ above $E_{F}$ for the relevant $\Gamma_{4}^{-}, \Gamma_{1}^{+}$state. The value is $5 \mathrm{eV}$ below the averaged $\Gamma_{4}^{-}, \Gamma_{1}^{+}$energy above $E_{F}$ in Ref. 8, and, however, agrees quite well with the energy position of prominent structures in both ARIPS [ $\simeq 20 \mathrm{eV}$ (Ref. 25 ), $\simeq 19 \mathrm{eV}$ (Ref. 26)]. (The calculations of Ref. 9 do not extend in this region of the conduction band and the conduction bands of Ref. 22 cannot be related unambiguously to bands of Ref. 8.)

In view of the above arguments, the (32 1 ) -eV peak or shoulder must be attributed to $\sigma \rightarrow \pi^{*}$ transitions, a part of which is marked by dashed arrows in Fig. 6(b). The calculated $\Gamma_{4}^{-} \rightarrow \Gamma_{3}^{+}, M_{1}^{+} \rightarrow M_{4}^{-}$, and $M_{2}^{-} \rightarrow M_{3}^{+}$transition energies, which are $31.3,30.5$, and $30.8 \mathrm{eV},{ }^{8}$ respectively, are not far from our measurement. Thus it is evident again that the weakly- $k_{z}$-dispersing states are much better represented by Ref. 8 than the strongly- $k_{z^{-}}$ dispersing ones.

\section{CONCLUSIONS}

We present the following conclusions.

(i) The dynamic structure factor, $S(\mathrm{q}, \omega)$, of HOP graphite, as measured with IXSS at $1 \mathrm{eV}$ resolution, is demonstrated to be crucially sensitive to details present in the band structure of graphite both in the interband transition and in the core-excitation regime. To obtain this result, transformation to $\epsilon_{2}(q, \omega)$ and the inclusion of both symmetry-induced selection rules and $q$ dependence of the TME's is required.

(ii) A maximum DOS in the first $\pi$-type conduction band at $1.5 \mathrm{eV}$ above $E_{F}$ could be established and was attributed to the range around $M_{4}^{-}$in agreement with practically all recent band-structure calculation. In the same manner a maximum DOS in the first $\sigma$-type conduction band at $9.5 \mathrm{eV}$ above $E_{F}$ was found and could be associated with bands near $M_{2}^{-}$, where their energy position is controversial among different band-structure calculations.

(iii) The occurrence of interband transitions into the recently postulated interlayer state of graphite was clearly established. Combining our results with valence-band photoemission data, the upper limit for the energy above $E_{F}$ of the $\Gamma_{1}^{+}$interlayer state was found to be $5.5 \mathrm{eV}$, in disagreement with some existing band-structure calculations, but in rather good agreement with other experimental informations of this band. In the same fashion, the existence of another presumably strongly- $k_{z}$ dispersing $\sigma$-type conduction band could be proved with some reliability. Its energy position above $E_{F}$ for $\Gamma_{4}^{-}, \Gamma_{1}^{+}$ is found to be $18.5 \mathrm{eV}$, contrary to theoretical predictions, but in reasonable agreement with high-DOS features of some experiments.

(iv) The energy of all other interband transitions deducible from our measurements and connected with weakly- $k_{z}$-dispersing states are in rather good agreement with recent calculations and other experiments.

(v) All band-structure information deduced from our measurements are bulk information, free from any influence of surface states or surface contaminations, since with IXSS the effective sample thickness is in the $\mathrm{mm}$ range, which makes IXSS best suited also for the study of intercalation compounds of graphite.

\section{ACKNOWLEDGMENTS}

We thank K. Fischer, A. Millhouse, R. Nusshardt, A. Kirfel, U. Dretzler, and G. Ernst for valuable help with the measurements at DORIS/HASYLAB (Hamburger Synchrotronstrahlungslabor, Deutsches Elektronensynchrotron). This work has been funded by the German Federal Minister of Research and Technology (BMFT) under Contract No. 05344 AX B2.
${ }^{1}$ P. R. Wallace, Phys. Rev. 71, 622 (1947).

${ }^{2}$ W. M. Lomer, Proc. R. Soc. London, Ser. A 277, 330 (1955).

${ }^{3}$ J. C. Slonczewski and P. R. Weiss, Phys. Rev. 109, 272 (1958).

${ }^{4}$ F. Bassani and G. Patori Parravicini, Nuovo Cimento B 50, 95 (1967).

${ }^{5}$ G. S. Painter and D. E. Ellis, Phys. Rev. B 1, 4747 (1970).

${ }^{6}$ H. Nagayoski, K. Nakao, and Y. Uemura, Solid State Commun. 10, 225 (1976).

${ }^{7}$ A. Zunger, Phys. Rev. B 17, 626 (1978).

${ }^{8}$ R. C. Tatar and S. Rabii, Phys. Rev. B 25, 4126 (1982).

${ }^{9}$ N. A. W. Holzwarth, S. G. Louie, and S. Rabii, Phys. Rev. B 26, 5382 (1982).

${ }^{10}$ M. Posternak, A. Baldereschi, A. J. Freeman, E. Wimmer, and M. Weinert, Phys. Rev. Lett. 50, 761 (1983).

${ }^{11}$ F. R. McFeely, S. P. Kowalczyk, L. Ley, R. G. Cavell, R. A. Pollak, and D. A. Shirley, Phys. Rev. B 9, 5268 (1974).

${ }^{12}$ A. Bianconi, S. B. M. Hagström, and R. Z. Bachrach, Phys. Rev. B 16, 5543 (1977).

${ }^{13}$ P. M. Williams, Nuovo Cimento 38B, 216 (1977).

${ }^{14}$ W. Eberhardt, I. T. McGovern, E. W. Plummer, and J. E. Fisher, Phys. Rev. Lett. 21, 200 (1980).

${ }^{15}$ A. R. Law, J. J. Barry, and H. P. Hughes, Phys. Rev. B 28, 5332 (1983).

${ }^{16}$ D. Marchand, C. Frétigny, M. Lagues, F. Batallan, Ch. Simon, I. Rosenman, and R. Pinchaux, Phys. Rev. B 30, 4788 (1984). 
${ }^{17}$ T. Takahashi, H. Tokailin, and T. Sagawa, Solid State Commun. 52, 765 (1984).

${ }^{18}$ A. R. Law, M. T. Johnson, and H. P. Hughes, Phys. Rev. B 34, 4289 (1986).

${ }^{19} \mathrm{G}$. Wiech and $\mathrm{W}$. Zahorowski, in Inner-Shell and X-Ray Physics of Atoms and Solids, edited by D. J. Fabian, H. Kleinpoppen, and L. M. Watson (Plenum, New York, 1981), p. 833.

${ }^{20}$ R. Chen, P. Trucano, and R. F. Stewart, Acta Crystallogr. Sect. A 33, 823 (1977).

${ }^{21}$ W. A. Reed, P. Eisenberger, K. C. Pandey, and L. C. Sayder, Phys. Rev. B 10, 1507 (1974); G. Loupias, J. Chomilier, and D. Guérard, Solid State Commun. 55, 299 (1985); S. Vasudevan, T. Rayment, B. G. Williams, and R. Holt, Proc. R. Soc. London, Ser. A 391, 109 (1984); R. Tyk, J. Felsteiner, I. Gertner, and R. Moreh, Phys. Rev. B 32, 2625 (1985).

${ }^{22}$ R. F. Willis, B. Fitton, and G. S. Painter, Phys. Rev. B 9, 1926 (1974).

${ }^{23}$ C. Mallet, J. Phys. C 14, L213 (1981).

${ }^{24}$ Th. Fauster, F. J. Himpsel, J. E. Fischer, and E. W. Plummer, Phys. Rev. Lett. 51, 430 (1983).

${ }^{25}$ H. Ohsawa, T. Takahashi, T. Kinoshita, Y. Enta, H. Ishii, and T. Sagawa, Solid State Commun. 61, 347 (1987).

${ }^{26}$ I. Schäfer, M. Schlüter, and M. Skibowski, Phys. Rev. B 35, 7663 (1987).

${ }^{27}$ A. R. Law, M. T. Johnson, H. P. Hughes, and H. A. Padmore, J. Phys. C 18, L297 (1985).

${ }^{28}$ L. Papagno and L. S. Caputi, Surf. Sci. 125, 530 (1983).

${ }^{29}$ L. S. Caputi, G. Chiarello, A. Santaniello, E. Colavita, and L. Papagno, Phys. Rev. B 34, 6080 (1986).

${ }^{30}$ A. Dittmar-Wituski, M. Naparty, and J. Skonieczny, J. Phys. C 18, 2563 (1985).

${ }^{31}$ D. Denley, P. Perfetti, R. S. Williams, and D. A. Shirley, Phys. Rev. B 21, 2267 (1980).

32J. F. Morar, F. J. Himpsel, G. Hollinger, J. L. Jordon, G. Hughes, and F. R. McFeely, Phys. Rev. B 33, 1346 (1986).

${ }^{33}$ B. M. Kincaid, A. E. Meixner, and P. M. Platzman, Phys. Rev. Lett. 40, 1296 (1978).

${ }^{34}$ E. J. Mele and J. J. Ritsko, Phys. Rev. Lett. 43, 68 (1979).

${ }^{35}$ V. Dose, G. Reusing, and H. Scheidt, Phys. Rev. B 26, 984 (1982).

${ }^{36}$ E. A. Taft and H. R. Philipp, Phys. Rev. 138, A197 (1965).

${ }^{37}$ E. Tosatti and F. Bassani, Nuovo Cimento 65B, 161 (1970).

${ }^{38}$ D. L. Greenaway and G. Harbeke, Phys. Rev. 178, 1341 (1969).

${ }^{39}$ L. G. Johnson and G. Dresselhaus, Phys. Rev. B 7, 2275 (1973).

${ }^{40}$ R. Klucker, M. Skibowski, and W. Steinmann, Phys. Status
Solidi B 65, 703 (1974).

${ }^{41}$ H. Venghaus, Phys. Status Solidi B 81, 221 (1977).

${ }^{42}$ K. Zeppenfeld, Z. Phys. 211, 391 (1968); 243, 229 (1971).

${ }^{43}$ H. Venghaus, Phys. Status Solidi B 71, 609 (1975).

${ }^{44}$ U. Büchner, Phys. Status Solidi B 81, 227 (1977).

${ }^{45}$ P. Eisenberger and P. M. Platzman, Phys. Rev. B 13, 934 (1976).

${ }^{46}$ P. Nozières and D. Pines, Phys. Rev. 113, 1254 (1959).

${ }^{47} \mathrm{D}$. Pines and P. Nozières, The Theory of Quantum Liquids (Benjamin, New York, 1966), Vol. 1.

${ }^{48}$ N. Wiser, Phys. Rev. 129, 62 (1963).

${ }^{49}$ H. Ehrenreich and M. H. Cohen, Phys. Rev. 115, 786 (1959).

${ }^{50}$ W. Schülke and H. Nagasawa, Nucl. Instrum. Methods 222, 203 (1984).

${ }^{51}$ W. Schülke, Nucl. Instrum. Methods A 246, 491 (1986).

${ }^{52}$ W. Schülke, H. Nagasawa, S. Mourikis, and P. Lanzki, Phys. Rev. B 33, 6744 (1986).

53J. Pirenne and E. Kartheuser, Physica (Utrecht) 20, 2004 (1964).

${ }^{54}$ C. A. Emeis, L. J. Oosterhoff, and Gonda de Vries, Proc. R. Soc. London, Ser. A 297, 54 (1967).

${ }^{55}$ Even if the dipole approximation breaks down, valuable information from core excitations in IXSS can be extracted as proposed by S. Doniach, P. M. Platzman, and J. T. Yue, Phys. Rev. B 4, 3345 (1971).

${ }^{56}$ E. Clementis and D. L. Raimondi, J. Chem. Phys. 38, 2686 (1963).

57J. C. Slater, Symmetry and Energy Bands in Crystals (Dover, New York, 1972).

58 J. W. Gadzuk and M. Šunjić, Phys. Rev. B 12, 524 (1975).

${ }^{59}$ A. Balzarotti, M. De Crescenzi, and L. Incoccia, Phys. Rev. B 25, 6349 (1982).

${ }^{60}$ S. M. Heald and E. A. Stern, Phys. Rev. B 17, 4069 (1978); A. D. Cox and J. H. Beaumont, Philos. Mag. B 42, 115 (1980); F. W. Kutzler, R. A. Scott, J. M. Berg, K. O. Hodgson, S. Doniach, S. P. Cramer, and C. H. Chang, J. Am. Chem. Soc. 103, 6083 (1981); D. H. Templeton and L. K. Templeton, Acta Crystallogr. Sect. A 41, 133 (1985).

${ }^{61}$ P. M. Platzman and P. Eisenberger, Phys. Rev. Lett. 33, 152 (1974).

${ }^{62}$ P. Eisenberger, W. C. Marra, and G. S. Brown, Phys. Rev. Lett. 45, 1439 (1980).

${ }^{63}$ W. Schülke, U. Bonse, H. Nagasawa, S. Mourikis, and A. Kaprolat, Phys. Rev. Lett. 59, 1361 (1987).

${ }^{64}$ M. Y. Chou, M. L. Cohen, and St. G. Louie, Phys. Rev. B 33, 6619 (1986). 